\title{
Moshe Zimmermann
}

\section{Strukturmerkmale der deutschen Geschichte - Deutsche Juden: Transterritoriale Kohärenzen}

Sucht man eine klare, eindeutige Zäsur in einem Sektor der deutschen Gesellschaft und der deutschen Geschichte des 20. Jahrhunderts, so findet man sie allein in der Geschichte der deutschen Juden: Das Jahr 1943 brachte das Ende des deutschen Judentums in Deutschland. Es war damit - anders als das Jahr 1945 - eine wirkliche Stunde Null; denn was sich als deutsches Judentum in der Zeitgeschichte nach 1943 und gewiß nach 1945 entwickelt hat, gehört in ein völlig neues Kapitel deutscher Geschichte bzw. jüdischer Geschichte in Deutschland, ist von neuem, ganz anderem Wesen.

Je stärker man davon ausgeht, daß im politischen, gesellschaftlichen und kulturellen Bereich der deutschen Geschichte das Jahr 1945 nicht ein totales Ende oder ein totaler Neuanfang war, je stärker das Bewußtsein für die Kontinuität der Strukturen und die in ihr enthaltenen Kohärenzen auch das Gefühl einer Zäsur zurücktreten läßt - wie es im allgemeinen in der neueren Forschungsliteratur geschieht, und wie es auch die Beiträge in diesem Band zeigen -, desto deutlicher wird die historiographische Bedeutung der Zäsur des Jahres 19431. Und in der intensiven Suche nach Strukturen und Kohärenz kann es dann sogar vorkommen, daß die Shoah unerwähnt bleibt - wie es hier auf unserer Konferenz geschah oder die Geschichte des deutschen Sports ohne Berücksichtigung des Ausschlusses von Juden aus dem Sportwesen geschrieben wird. Insbesondere auf dem Hintergrund des Versuchs, den eigentlichen Bruch der deutschen Geschichte des

1 Ein symptomatischer Fall: Klaus Bade, Integration, Naturalization and Ethno-National Traditions in Germany: From the Citizenship Law of 1913 to the Law of 1999, in: Larry E. Jones (Hrsg.), Crossing Boundaries. The Exclusion and Inclusion of Minorities in Germany and the United States (New York 2001) 29-49. Hier wird von "intrinsic coherence of ethnonational thinking and migration policies" während der gesamten Periode gesprochen. Auch die Aufnahme von Juden aus den Ländern der ehemaligen Sowjetunion in den 1990er Jahren und sogar die NS-Politik passen in diese Kohärenz hinein: „In the history of transnational migration, citizenship rights and related policies, the Nazi period introduced a new dimension, different in quality yet still representing an important line of continuity with the past" (ebd. 38; Hervorhebung M.Z.). In diesem Fall wird die Kohärenz keinesweg verharmlosend oder relativierend im Sinne einer revisionistischen Geschichtsschreibung funktionalisiert, aus der Perspektive der deutsch-jüdischen Geschichte scheint diese Kohärenz jedoch zumindest überzogen zu sein. 
20. Jahrhunderts von den 1930er und 1940er Jahren in die 1970er Jahre zu verlagern, kann die gesamte, von der Strukturgeschichte angebotene Vorstellung von Kohärenz aus der Perspektive der deutsch-jüdischen Geschichte sinnvoll hinterfragt oder überprüft werden.

Ausgehend von der entscheidenden Zäsur des Jahres 1943 öffnen sich der Behandlung des gestellten Themas im vorliegenden Rahmen zwei relevante Zugänge: Zum einen bietet sich auf der Suche nach eventueller Kohärenz oder möglichen Zäsuren der Zugang der vergleichenden Betrachtung der drei, dem Ende gewissermaßen unmittelbar vorangegangenen Epochen der Geschichte dieses Segments der deutschen Gesellschaft - des Kaiserreichs, der Weimarer Republik und des „Dritten Reiches“. Zum anderen kann versucht werden, durch einen Akt der Verlagerung die absolute Zäsur des Jahres 1943 zu überwinden oder zu relativieren, um eine Kohärenz über die Jahre 1943-1945 hinaus herzustellen. Diese Verlagerung wiederum kann auf zweierlei Weise erfolgen: zum einen durch die schwerpunktmäßige Analyse des kulturellen Sektors anstatt des konkreten sozio-politischen Bereiches (so zeigt das Phänomen des Rassismus zum Beispiel seine Kohärenz auch jenseits des Antisemitismus, der nach 1945 an Relevanz verlor); zum anderen - und dies ist nicht unbedingt vom ersten Ansatz zu trennen - durch die Betrachtung deutscher Juden, die ihr Leben als solches nicht in der Shoah verloren haben, sondern in andere Länder emigrieren konnten, in denen sie die Selbstdefinition als deutsche Juden aufrechterhalten haben.

\section{Von der Kohärenz der deutsch-jüdischen Geschichte vor 1943}

Aus der Sicht von sogenannten „Assimilanten“, Nationaljuden oder auch von Antisemiten scheint die deutsch-jüdische Geschichte bis 1933 eine klare Kohärenz zu zeigen. Im Mittelpunkt der historischen Analyse steht in der Regel der Begriff der Assimilation - und die Geschichte des deutschen Judentums bzw. der deutschen Juden gilt als paradigmatisch für den Prozeß der Assimilation.

Nachdem das deutsche Reich 1871 der jüdischen Bevölkerung die Emanzipation, also die politische und juristische Gleichberechtigung, gewährt hatte, kreiste die Selbstdarstellung, vor allem aber die Kritik inner- und außerhalb des deutschen Judentums, um die Frage der Assimilation. Die Historiographie sprach bisweilen auch von Integration, Akkulturation und Amalgamation, griff zu den provokanten Begriffen der Symbiose, der Inklusion und Exklusion: oder redete über „Outsider und Insider". Trotz dieser variablen Terminologie blieb das historische Problem jedoch im großen und ganzen weiterhin die Frage der Assimilation, also der Anpassung einer Minderheitsgruppe an ihre Umgebung. Daß Zygmunt Bauman in seinem Buch „Modernity and Ambivalence" 2 dem Begriff der Assimilation, auch mit Blick auf das Schicksal des Judentums, so viel Aufmerksamkeit geschenkt hat, trug dazu bei, die Diskussion in ihre altbekannte Begrifflichkeit zu-

2 Zygmunt Bauman, Modernity and Ambivalence (Ithaca 1991). 
rückzuführen. So behandelt man heute wieder zunehmend die Kohärenz des "Projekts des Assimilation“ oder auch des "Traums der Assimilation“ als Kern der Geschichte deutscher Juden ${ }^{3}$. Vor allem aber aus der Sicht eines Historikers, der sich der hebräischen Wörter hitbollelut und tmi'ab bewußt ist, bleibt der Begriff der „Assimilation“ unangefochten.

Baumans Angriff auf die nivellierende Funktion der Assimilation, bzw. die Unterdrückung von Differenzen innerhalb der Gesellschaft, war nicht nur Schützenhilfe für traditionelle Assimilationskritiker, für die Verteidiger von „Differenz“ in der liberalen Gesellschaft, sondern auch für diejenigen, die die historische Diskussion um das Schicksal der deutschen Juden zum vertrauten Diskurs zurückführen wollten, selbst wenn dafür der Preis der analytischen „Unsauberkeit“4 dieses Begriffs zu zahlen war. Ein Beispiel dafür, wie „unsauber“ bzw. verwirrend der Begriff der Assimilation sein kann, kommt in einem nur wenige Jahre alten Beitrag über Assimilation und Kohärenz in der Geschichte der deutschen Juden seit dem Ende des 19. Jahrhunderts zum Ausdruck, der ausgerechnet den jüdischen Selbsthaß als „Form einer vollständigen Assimilation“ werten möchte ${ }^{5}$. Allerdings ist das Konzept der Assimilation als analytisches Instrument keineswegs ungeeigneter als die genannten konkurrierenden Begriffe.

Möglicherweise erweist sich die Kohärenz jüdischer Geschichte am stärksten in den historischen, juristischen, politischen und historiographischen Versuchen, zunächst einmal den Charakter der als Juden/deutsche Juden/deutsches Judentum bezeichneten Gruppe zu definieren, um dann im Anschluß den Begriff der Assimilation besser mit Konturen versehen zu können. Die Frage, was diese Menschengruppe ausmacht, war und bleibt Gegenstand der Diskussion von Juden (wie auch immer "Jude“ inhaltlich zu füllen sein mag), Nichtjuden, Zeitgenossen und Historikern zugleich: Religion? Konfession? Schicksalsgemeinschaft? Moderne? Nation? Rasse? Außenseiter ${ }^{6}$ ? Sozialmilieu? Sozialmoralisches Milieu? Ethnische Gruppe ${ }^{7}$ ? Die letztgenannten Begriffe beherrschen die historiographische Diskussion der letzten Jahrzehnte, treten in der Regel auch neben die Hilfsbegriffe der Exklusion/Inklusion ${ }^{8}$, führen jedoch im Endeffekt (und nicht nur in

3 Michael Brenner, Derek J. Penslar (Hrsg.), In Search of Jewish Community. Jewish Identities in Germany and Austria 1918-1933 (Bloomington 1998) Einleitung xii; im folgenden zitiert: Brenner, Penslar, Jewish Community; sowie Shulamit Volkov, German Jews between Fulfillment and Disillusion, in: Brenner, Penslar, Jewish Community 11.

4 Shulamit Volkov, Jüdische Assimilation und Eigenart im Kaiserreich, in: Geschichte und Gesellschaft 9 (1983) 331-348.

5 Panikos Panayi, Continuities and Discontinuities in Race: Jews, Gypsies and Slavs under the Weimar Republic and the Third Reich, in: Panikos Panayi (Hrsg.), Weimar and Nazi Germany. Continuities and Discontinuities (Harlow 2001) 222.

6 Vgl. Mattbias Hambrock, Die Etablierung der Außenseiter. Der Verband nationaldeutscher Juden 1921-35 (Köln 2003) insbesondere Kap. 2; im folgenden zitiert: Hambrock, Außenseiter.

7 Vgl. Till van Rabden, Juden und andere Breslauer (Göttingen 2000) 20f.; im folgenden zitiert: van Rabden, Juden.

8 Niklas Luhmann, Inklusion und Exklusion, in: Helmut Berding (Hrsg.), Nationales Bewußtsein und kollektive Identität (Frankfurt a.M. 1994) $24 \mathrm{ff}$. 
der Folge Zygmunt Baumans) zurück zum Ausgangspunkt: zur Assimilation. Welche Aussagekraft hat denn zum Beispiel schon die Feststellung, daß deutsche Juden anders als Katholiken keine eigene Partei und keine Abschottung gegen die Teilnahme am Sozialnetz, an Kultur und Medien der nichtjüdischen Gesellschaft praktizierten ${ }^{9}$, wenn nicht die, daß hier der Prozeß der Assimilation effektiv abgelaufen ist. Allerdings relativiert der Historiker der jüdischen Geschichte selbstverständlich die Gruppendefinition, weil man in der Moderne wie in der Postmoderne nicht von einem homogenen Judentum ausgehen kann, sondern mit einer pluralistischen, vielfältigen Vorstellung von Judentum und Juden als Gesamtheit das Problem angreifen muß.

Der Prozeß der Anpassung an die Umgebung, der im Zeitalter des Absolutismus und der Aufklärung im Zeichen des Nebeneinanders von zwei religiösen Gemeinden mitten im deutschen Partikularismus stand, mußte mit der Verlagerung des Schwerpunkts des allgemeinen politischen Diskurses vornehmlich mit dem populären Schlagwort „Nation“ geführt werden. Vor der Erfindung der jüdischen Nation, also vor dem Ende des 19. Jahrhunderts, schienen Anpassung und Assimilation unproblematisch gewesen zu sein, unproblematischer jedenfalls als vor der Säkularisierung der Beziehungen zwischen Juden und ihrer Umgebung ${ }^{10}$. In dieser Hinsicht unterschied sich allerdings das deutsche Judentum kaum vom Judentum in anderen westlichen oder mitteleuropäischen Staaten und Gesellschaften.

Mehr noch: Die Erfindung der Nation oder die Verwendung des Begriffs der Nation half Juden zunächst die alten, konfessionsbedingten Markierungen zwischen Juden und Nichtjuden zu überbrücken oder zu vertuschen. In der Nation, in unserem Falle der deutschen Nation, aufzugehen, deutscher Staatsbürger mosaischen Glaubens zu sein, schien die eleganteste Antwort auf die sogenannte „Judenfrage“ zu sein. Die Geschichte der deutschen Juden im Kaiserreich, vor allem im Wilhelminischen Zeitalter, kommt dem Leser der Geschichte der deutschen Juden eher wie eine bloße Beschreibung des Prozesses der Assimilation in die national-deutsche Gesellschaft vor, ein Prozeß, der sich dann in den nächsten Epochen - mindestens bis 1933 - fortgesetzt hat.

Bis heute ist die „Kunstwart"-Debatte des Jahres 1912 ein Meilenstein in der Diskussion um den Erfolg der Nationalisierung, sprich: der Assimilation, der Juden im deutschen Kaiserreich unter dem Druck der Modernisierung. Moritz Goldsteins Aufsatz „Deutsch-jüdischer Parnaß“ löste die Debatte aus, ob Juden nun "ganz deutsch" oder "ganz undeutsch" geworden bzw. geblieben sind oder ob sie vielleicht sogar über das Ziel hinausgeschossen sind in ihren Bemühungen um Assimilation und Integration in das „Deutschtum“ ${ }^{11}$. Nicht nur die assimila-

9 Van Rabden, Juden $19 \mathrm{f}$.

10 Vgl. dazu das Vorwort in Moshe Zimmermann, Hamburgischer Patriotismus und deutscher Nationalismus. Die Emanzipation der Juden in Hamburg 1830-1865 (Hamburg 1979) 12-15.

11 Moritz Goldstein, Deutsch-Jüdischer Parnaß, in: Der Kunstwart (März 1912) 281-294; Steven E. Ascbbeim, 1912: The publication of Moritz Goldstein's "The German-Jewish Par- 
torische oder anti-assimilatorische Antwort, sondern auch die Fragestellung blieb kohärent - sie war für deutsche Juden und für ihr gesellschaftliches Umfeld bis in die Zeit des Nationalsozialismus hinein eine Frage nach der kollektiven Identität, und sie begleitet seither den Historiker und Laien gleichermaßen.

Weil eben die Nation - die deutsche Nation - den Zeitgenossen und Historikern als primärer Rahmen des Assimilationsprozesses galt, schaute man in der Regel darüber hinweg, daß damit im Grunde eine Assimilation an das bürgerliche Verständnis von Nation gemeint war, daß es um die Anpassung an die spezifischen Werte und Anschauungen des Bürgertums bzw. "die Angleichung der ,nicht bürgerlichen 'Schichten an das Ideal des Bürgertums", an die bürgerliche Lebensführung ging12. Man konnte daher auch von "Überanpassung“ sprechen, wofür es, nach George Mosse, auch einen Preis gab - die „Entfremdung von den deutschen Massen“13.

Noch kritischer formuliert ein junger Historiker den Vorgang: „Die Idee der Assimilation als der Heraufführung der Juden zu einem weltbürgerlichen Humanismus verkam unter den Liberalen dann in der zweiten Hälfte des 19. Jahrhunderts zu der Frage, inwieweit die Juden sich an den deutschen Nationalstaat anpassen können." 14 Und eben weil es der Liberalismus war, der diese Forderung nach Assimilation stellte, galt der Mehrheit deutscher Juden, die im Liberalismus den Garanten ihrer Rechte sahen und vornehmlich liberale Parteien wählten ${ }^{15}$, diese Vorstellung als Unterpfand ihrer Akzeptanz als gleichberechtigte Bürger vor und auch noch nach 1933. Daß die Teilnahme an der nationalen Gesellschaft nicht nur - und vor allem nicht mehr - auf liberalem Fundament ruhte, versuchte man dabei zu ignorieren oder zu verdrängen. Das Eigentliche blieb jedoch bis zum bitteren Ende die Verbundenheit mit der „deutsch-bürgerlichen Kultur“ 16 , mit dem bürgerlichen Bildungsideal, also letztlich mit Elementen, die keineswegs identisch sind mit Deutschsein oder der deutschen Gesellschaft im allgemeinen. Auf einer entsprechenden Ebene wurde dann auch die Diskussion zwischen Juden und Nichtjuden ausgetragen: Wenn man Juden seit 1871 mangelnde Assimilationsbereitschaft zum Vorwurf machte, dann standen die "deutschen“, nicht die

nassus “ sparks Debate over Assimilation etc., in: Sander L. Gilman, Jack Zipes (Hrsg.), The Yale Companion to Jewish Writing and Thought in German Culture (New Haven 1997) 299-305; Noah W. Isenberg, Between Redemption and Doom. The Strains of German-Jewish Modernism (Nebraska 1999) $8 \mathrm{ff}$.

12 Hans-Joachim Salecker, Der Liberalismus und die Erfahrung der Differenz (Bodenheim 1999) 181, 184; im folgenden zitiert: Salecker, Liberalismus; vgl. auch Jürgen Kocka (Hrsg.), Bürger und Bürgerlichkeit im 19. Jahrhundert (Göttingen 1987) 21-63.

13 George L. Mosse, German Jews beyond Judaism (Bloomington 1985) 8; im folgenden zitiert: Mosse, Beyond Judaism.

14 Salecker, Liberalismus 202, 205.

15 Martin Liepach, Das Wahlverhalten der jüdischen Bevölkerung: Zur politischen Orientierung der Juden in der Weimarer Republik (Tübingen 1996); Manfred Hettling, Sozialstruktur und politische Orientierung im Kaiserreich, in: Manfred Hettling u. a. (Hrsg.), In Breslau zuhause? (Hamburg 2003) 113-130.

16 Jost Hermand, Judentum und deutsche Kultur (Köln 1996) 146; im folgenden zitiert: Hermand, Judentum. 
universalen Werte im Mittelpunkt. Und so kann es nicht verwundern, daß die Antwort der Juden auch nach 1933 diese Linie weiterverfolgte. Selbst wenn im nachhinein in einer postmodernen Geschichtsschreibung die Nation und der $\mathrm{Na}$ tionalismus dekonstruiert werden - wie in Volkovs Ansatz ${ }^{17}$-, so ändert dies am Bewußtsein und an der Wahrnehmung deutscher Juden vor 1943 nichts. Es war dann eben das Jahr 1943, in dem, wie erwähnt, die tiefste Zäsur in der Geschichte dieses Sektors der deutschen Gesellschaft eintrat; und daher entsteht in der zweiten Hälfte des 20. Jahrhunderts - anders als in der angeblich so kohärenten „allgemeinen" deutschen Geschichte - auch keine Diskrepanz zwischen einer vermeintlichen Kohärenz in der Geschichte der deutschen Juden nach 1943 (die es ja gar nicht mehr gab) und einer neuen, postmodernen Geschichtsschreibung, die sich auf das gesamte Jahrhundert beziehen möchte und dabei Kohärenzen zerstört. Man muß ja eine Kohärenz nicht in Frage stellen, wenn sie nicht von Beginn an vermutet wurde.

Oft erhielt das Konzept der Assimilation eine radikale Auslegung, denn auch in der angeblich säkularisierten Gesellschaft erwartete man nur allzu häufig die Taufe als den "Abschluß" der Assimilation. Daher war es schwierig, über Stufen oder „Erfolg“ des Prozesses zu sprechen. Ist man erst mit der Taufe oder mindestens mit dem Austritt aus jeglicher jüdischen Vereinigung wirklich assimiliert, dann reichen andere Maßnahmen nicht aus, um als assimiliert oder integriert gelten zu können. Man sah sich also quasi automatisch mit dem Vorwurf konfrontiert, "nicht genügend" assimiliert zu sein. Gershom Scholem, ein scharfer Kritiker der assimilationsfreudigen "Wissenschaft des Judentums" und der These von der "deutsch-jüdischen Symbiose“, meinte zwar aus seiner persönlichen Erfahrung abschließend einerseits, die „Assimilation ging sehr weit“, sprach andererseits aber immer wieder von "Selbstbetrug" oder „Blindheit" der deutschen Juden, die an die Assimilation und Integration in die deutsche Gesellschaft glaubten, sie aber in der Praxis nicht erleben durften. Für Scholem lag die eigentliche Grenze zwischen vollendeter und nicht vollendeter Assimilation im gesellschaftlichen Verkehr mit der nichtjüdischen Umgebung. Sogar sein Vater, den er als einen ausgesprochenen „Protagonisten der Assimilation“ bezeichnete, war gegen die "Mischehe“, und Besucher und Freunde der Eltern waren ,ausschließlich Juden"18. Kurz: Für Scholem und viele Zionisten waren - wie auf der anderen Seite, aber aufgrund anderer Kriterien, für Antisemiten - die deutschen Juden nicht im vollen Sinn des Wortes assimiliert. Die Kohärenz ist nach dieser Auslegung die Kohärenz eines nur scheinbar vollzogenen Prozesses der Assimilation, der Diskrepanz zwischen dem Streben, assimiliert zu sein, und der Realität der Unerreichbarkeit dieses Ziels.

17 Shulamit Volkov, Minderheiten und Nationalstaat. Eine postmoderne Perspektive, in: Shulamit Volkov, Das jüdische Projekt der Moderne (München 2001) 13-31.

18 Gershom Scholem, On the Social Psychology of the Jews in Germany: 1900-1933, in: David Bronson (Hrsg.), Jews and Germans from 1860 to 1933: the Problematic Symbiosis (Heidelberg 1979) 9-32. 
Bei Scholem stellt sich, wie überhaupt allgemein, die Frage nach einer möglichen "Vollständigkeit“ des Assimilationsprozesses. Wenn man von „Vollinklusion“" sprechen darf, was soll dann „vollständige Assimilation“ heißen? Kann Assimilation ein Prozeß auf Raten sein, von dem man am Ende unbedingt eine Vollständigkeit erwarten darf? Die retrospektive Kritik an der Assimilation betont ja geradezu die Schädlichkeit der Idee vom „Schmelztiegel“ und von der Gleichschaltung, also einer „vollständigen“ Assimilation.

Geht man von einer facettenreichen oder pluralistischen Gesellschaft aus, ist die Idee einer „vollständigen“ Assimilation entweder unpraktizierbar oder unakzeptabel. Der Historiker, der "Assimilation“ nicht mit dem Verlangen nach totaler Gleichschaltung verwechselt, muß sich mit einiger Berechtigung in den Kontext des Wahrnehmungshorizontes der meisten deutschen Juden in der Zeit vor der großen Zäsur hineinversetzen. Dabei sollte auch zwischen dem Adjektiv „assimiliert" und dem Schimpfwort „Assimilant“ unterschieden werden. George Mosse hat einen wichtigen Beitrag zur Lösung des konzeptuellen Problems geleistet, indem er vom „Ausfüllen“ der Lücke zwischen Judentum und traditionellem Christentum durch Bildung sprach ${ }^{19}$. Der deutsche Begriff der Bildung umfaßt demnach alle Elemente der Assimilation, die Juden aus dem herkömmlichen Rahmen heraus in die moderne Gesellschaft hineinführen, ohne am Ende zum Christentum übertreten $\mathrm{zu}$ müssen. Bildung schafft gewissermaßen Assimilation ohne Taufe. Und eben dies praktizierten deutsche Juden - die einen mehr, die anderen weniger.

Wer Assimilation als analytischen Kernbegriff benutzt, begreift das Revolutionsjahr 1918 nur als Vertiefung und Beschleunigung des Assimilationsprozesses. Während Antisemiten und etliche Nostalgiker die Weimarer Republik als „verjudet" bezeichneten, war der nach 1918 geschaffene Rahmen ideal für die Intensivierung der Assimilation. Von einer "Judenrepublik" oder einer "Judenkultur" zu sprechen, also von einer umgekehrten Assimilation, ist auf jeden Fall irreführend ${ }^{20}$. Die Assimilation kam vielfältig zum Ausdruck, weil die Gesellschaft insgesamt pluralistische Ausdrucksformen gewonnen hatte. Juden konnten sich im liberalen, im revolutionären oder konservativen Umfeld assimilieren. Für orthodoxe oder zionistische Juden war der Begriff der Assimilation zwar ein Schimpfwort. Aber sogar in den Augen Martin Bubers, der ebenfalls Zionist war, lag die eigentliche Gefahr nicht in der Assimilation, sondern in der Atomisierung der jüdischen Gesellschaft ${ }^{21}$, also im Verlust der Gemeinschaftsbindungen in einer sich modernisierenden Gesellschaft. Weil Assimilation letztlich - und darauf soll später noch einmal eingegangen werden - nicht unbedingt im $W_{1}$ derspruch zu einem „jüdischen Bewußtsein“" stand, konnte man sogar als Zionist oder orthodoxer Jude mehrere Faktoren der Assimilation bewußt oder unbewußt akzeptieren.

19 Mosse, Beyond Judaism 42.

20 Hermand, Judentum 158.

21 Martin Buber, Die Losung, in: Der Jude 1 (1916). 
Mit dem Aufstieg zur Macht versuchten die Nationalsozialisten zunächst die Emanzipation der Juden in der Absicht rückgängig zu machen, den Prozeß der Assimilation umzukehren. Die Emanzipation galt den Antisemiten als schädliches Instrument der Assimilation, die wiederum als die größte Gefahr für die „Volksgemeinschaft “, d.h. die völkisch verstandene Nation, und ihre Kultur begriffen wurde. Somit hatte die Aufhebung der Emanzipation ein Ziel - die Entfernung der Juden aus der Gesellschaft, also radikalste Dissimilation. Ismar Elbogen faßte mit Bedauern in seiner 1935 erschienenen "Geschichte der Juden in Deutschland“ (nach offizieller Anordnung konnte er nicht mehr von deutschen Juden sprechen) zusammen: Das Regime „schloß die Juden von der Volksgemeinschaft aus“, und „die seit der Zeit der Aufklärung geforderte Assimilation der Juden wurde verworfen“22. Allerdings konnte auch das „Dritte Reich“ die Assimilation nicht so schnell und abrupt beseitigen, wie mancher es gewollt hätte: Die in Deutschland verbliebenen Juden konnten zwar unter der rassistischen Gesetzgebung - vom Gesetz zur Wiederherstellung des Berufsbeamtentums vom April 1933 bis zu den Nürnberger Gesetzen vom September 1935 und anderen späteren Maßnahmen die physische und soziale Trennung nicht bekämpfen. Auch wurde ihre Aktivität im Kulturbereich im Rahmen des „Kulturbundes" 23 gettoisiert. Doch die Haltung, die Mores, die Mentalität standen weiterhin im Zeichen der Assimilation. Schon die Tatsache, daß auf die diskriminierenden Gesetzgebungsmaßnahmen keine Massenauswanderung erfolgt war, zeigt, daß sogar das Jahr 1933 der Assimilation kein abruptes Ende bereitet hat. Man war getrennt und doch assimiliert. Man glaubte nach einer bestimmten Logik konsequent und kohärent noch immer an eine mögliche Fortsetzung der Assimilation. Dies zeigten die Agenden des Central Vereins, des Reichsverbands jüdischer Frontsoldaten und ganz besonders deutlich des als exotisch erscheinenden, kleinen Verbandes nationaldeutscher Juden nach 1933. „Der Grundgedanke der ,nationalen Erhebung', wie ihn die Organisation sah, nämlich die Verwirklichung des Ideals einer ,deutschen Volksgemeinschaft", wurde ausdrücklich als eine große Aufgabe bejaht", ist das Fazit des Historikers dieses Verbandes. In den Augen des Regimes galt das sogar als Versuch, „eine Art NSDAP der Juden [zu] sein" 24 . Diese Tendenz war - in abgemilderter Form - jedoch nicht allein für den Verband nationaldeutscher Juden charakteristisch. Und sie war nicht nur in der Zeit unmittelbar nach der „Machtergreifung" der Nationalsozialisten spürbar: Noch zwei Jahre später erschreckte die Berliner Landespolizei vor der „sogenannte[n] nationaldeutsche[n] Richtung“ des von den Nationalsozialisten selbst geschaffenen „jüdischen Kulturbundes“, als man dort „die jüdische Einsatzbereitschaft für deutsche Belange "versicherte und sich auf die "Verbundenheit mit dem deutschen Volk“ berief. Juden versuchten auch, weiterhin "Propaganda für das Verbleiben in Deutschland“ zu machen,

22 Ismar Elbogen, Geschichte der Juden in Deutschland (Berlin 1935) 313.

23 Yehoyakim Kochavi, Bewaffnung für eine nationale Existenz (hebr.; Lochamei Hagetaot 1988).

24 Hambrock, Außenseiter 571, 656. 
wollten die Hakenkreuzfahne hissen und bestanden darauf, im Rahmen des neuen Wehrgesetzes eingezogen zu werden. Selbst nach dem Erlaß der Nürnberger Gesetze war diese Tendenz nicht gänzlich verschwunden ${ }^{25}$.

Der Assimilationsprozeß resultiert nicht nur aus der Gegenüberstellung von Individuum und Gruppe, sondern entspringt auch dem Gegenüber von zwei Menschengruppen. Daher tritt das Paradox von "getrennt und assimiliert zugleich" noch stärker aus einem anderen Blickwinkel hervor: Was sich nach eigener Vorstellung als Gegenwehr gegen die Assimilation präsentierte, nämlich der jüdische Nationalismus, vor allem der Zionismus, war in gewisser Weise ein Akt der kollektiven Assimilation. Man paßte sich an die nationale Denkweise und Einstellung der Umgebung an und erfand eine Alternative zum Anschluß an die deutsche Nation bzw. eine Antwort auf Versuche des Ausschlusses der Juden aus dem Kollektiv der deutschen Nation - die Definition des Judentums als Nation. Schritte und Maßnahmen, die auf die Herausbildung einer jüdisch-nationalen Gruppe hinausliefen, waren also Akte der Assimilation an die Denkweise der national denkenden Umgebung. Schaut man auf die Mythen und Riten der jüdischen Nation, so ist die Anpassung und Nachahmung der umgebenden Nation auffällig. Diese kollektive Haltung und Übernahme von Denkmustern in die jüdische $\mathrm{Na}$ tionalbewegung stand allerdings der individuellen Assimilation nicht im Wege, wie die Bereitschaft auch von Zionisten, für Deutschland 1914 in den Krieg zu ziehen, demonstrierte.

Themen wie Geschichte und Nation, Sprache und Nation oder „körperliche Regeneration“ als Vorbedingung für die Verwirklichung der Nation - das alles konnte man in einer jüdischen Variante des deutschen Originals finden. Die Rolle des Turnens im Zionismus läßt keinen Zweifel aufkommen, wo der Ursprung dieser jüdisch-nationalen Tugend lag. Max Nordau oder andere, die sich diesbezüglich in der deutsch-zionistischen Literatur zu Wort meldeten, haben auf diesen Zusammenhang auch offen hingewiesen. Robert Weltsch verglich in diesem Zusammenhang „die Lage des deutschen Volkes vor 1813 mit der Lage des Judentums von heute" 26 . Daß dieser Prozeß der kollektiven Assimilation zu mancher paradoxen Situation führte, zeigt nicht nur die Zeit vor, sondern auch nach 1933. Ein extremes Beispiel: Ernst Moritz Arndts Lied „Der Gott der Eisen wachsen ließ" wurde ins Hebräische übertragen und vom rechten Flügel des Zionismus während des Zweiten Weltkriegs für seine Ziele im Kampf gegen die arabische Nationalbewegung und die britische Mandatsherrschaft gleichermaßen eingesetzt.

Die Kohärenz der Geschichte der deutschen Juden alleine unter dem Begriff der Assimilation zu subsumieren, wäre selbstverständlich übertrieben und einseitig. Der Begriff der Moderne, so wie ihn Shulamit Volkov anbietet, ist sicherlich ein

25 Hambrock, Außenseiter 660-679.

26 Robert Weltsch, 1813, in: Jüdische Monathefte für Turnen und Sport (März 1913) 46-48; vgl. auch Moshe Zimmermann, Die Antike als Erinnerungsarsenal: Vorbilder des jüdischen Sports, in: Yotam Hotam, Joachim Jakob (Hrsg.), Konstruktionen von Erinnerung im deutschen Judentum und nach der Emigration (Göttingen 2003). 
weiterer sinnvoller Rahmen. Nur beschreibt dieser Begriff, dessen Konturen auch nach intensiver wissenschaftlicher Behandlung unklar bleiben, Prozesse, die nicht nur für die jüdische Gesellschaft spezifisch waren. Spezifische, auf die jüdische Gesellschaft abzielende Begriffe waren vielmehr „Untergang“ oder „Renaissance“. Mit ihnen wird eine Kohärenz beschrieben, die selbst bis in die Zeit nach 1933 hinüberreichte und zu einer Relativierung der meist als Krisen oder Zäsuren bezeichneten Ereignisse von 1918 und gar 1933 führt. Beide Begriffe dienten Zeitgenossen und Historikern gleichermaßen als Orientierungshilfe und Instrumente der Wahrnehmung. So widersprüchlich beide Begriffe auch sein mögen, sie haben sich dialektisch ergänzt und eine paradoxe Kohärenz geschaffen. Ernst Simon nannte sein Buch „Aufbau im Untergang “27; aber der Titel „Renaissance im Untergang" wäre sicher nicht nur Simon, sondern auch vielen anderen Beobachtern als geeignet erschienen, um die Zeit vor (!) und nach 1933 zu beschreiben.

Parallel zum Optimismus, der das reale oder vermeintliche Vordringen der Assimilation begleitete, verbreitete sich als Kontrapunkt auch eine Untergangsstimmung. Vielleicht war es der Kulturpessimismus des Wilhelminischen Zeitalters, der hier „ansteckend“ gewirkt hatte, vielleicht aber auch die Folge der Auseinandersetzung mit dem modernen Antisemitismus, der den Weg der Assimilation mit Hilfe von völkischen und rassistischen Parolen zu blockieren drohte. Da der Antisemitismus ein Merkmal der nichtjüdischen, nicht der jüdischen Gesellschaft ist und er in diesem Zusammenhang nur dann relevant ist, wenn er eine Reaktion von jüdischer Seite hervorruft, ist die intensiv erforschte Frage der Kohärenz und Kontinuität des deutschen Antisemitismus ${ }^{28}$ allein eine sekundäre Frage im $\mathrm{Zu}$ sammenhang des vorliegenden Beitrags. Die Geschichte des deutschen Antisemitismus ist demnach nur insofern ein Abschnitt der deutsch-jüdischen Geschichte, als das Schicksal von Juden kulturell, gesellschaftlich und politisch vom Antisemitismus beeinflußt wurde. Zur Untergangsstimmung hat der Antisemitismus sicher beigetragen, bis 1932 jedoch keineswegs in entscheidendem Maße. Das beweisen auch die marginalen Zahlen der jüdischen Auswanderer bis zu diesem Jahr. Die viel diskutierte, düstere Prognose in Felix Theilhabers Buch "Der Untergang der deutschen Juden " 29 von 1912 stand eher im Zusammenhang mit demographischen Angaben zu den Bereichen der Eheschließungen, der Familienplanung und der wirtschaftlichen Umstrukturierung - also mit Merkmalen der Assimilation - als mit dem Druck des Antisemitismus. Als Otto Heller genau zwanzig Jahre später seinen „Untergang des Judentums“ 30 schrieb, verstand er darunter eine proleta-

27 Ernst Simon, Aufbau im Untergang. Jüdische Erwachsenenbildung im nationalsozialistischen Deutschland als geistiger Widerstand (Tübingen 1959).

28 Dazu u. a. Richard S. Levy, The Downfall of the Anti-Semitic Political Parties in Imperial Germany (New Haven 1975); Moshe Zimmermann, Aufkommen und Diskreditierung des Begriffs ,Antisemitismus', in: Ursula Büttner (Hrsg.), Das Unrechtsregime, Bd. I: Ideologie Herrschaftssystem - Wirkung in Europa (Hamburg 1986) 59-77; sowie Shulamit Volkov, Antisemitismus als kultureller Code (München 2000).

${ }^{29}$ Felix Theilhaber, Der Untergang der deutschen Juden (Berlin 1912).

30 Otto Heller, Der Untergang des Judentums. Die Judenfrage, ihre Kritik, ihre Lösung durch den Sozialismus (Wien 1931). 
risch-revolutionäre Lösung der Judenfrage. In diese Richtung ging auch Alfred Kantorowicz' Buch „Liquidation der Judenfrage“ von $1932^{31}$, in dem die Sowjetunion als Beispiel herangezogen wurde. Die Untergangsstimmung, die die letzte Phase der Weimarer Republik und die erste Zeit nach der Machtübernahme der Nationalsozialisten kennzeichnete, beruhte somit auf einer vorgegebenen Stimmungsbasis. Seit 1933 wurde der Untergang allerdings vom Staat in Form einer anti-assimilatorischen Politik oktroyiert und beschleunigt. Fortan „hinkte“ die Wahrnehmung des Untergangs der Realität hinterher, nicht umgekehrt ${ }^{32}$.

Der Begriff der Renaissance ist im deutsch-jüdischen Kontext unauflöslich mit der Frage der Dissimilation verbunden ${ }^{33}$. Noch vor Beginn des 20. Jahrhunderts bezeichneten Zeitgenossen die Abkehr von der Idee der Assimilation als Dissimilation. Eine derartige Abkehr aber wurde sowohl von Judenfeinden als auch von Nationaljuden (Zionisten) begrüßt. In der Geschichtsschreibung nach dem Zweiten Weltkrieg wurde dann systematisch nach den Elementen der jüdischen Kollektividentität und Aktivität gefragt. Derartige Elemente einer Dissimilation, die man als Gegenbewegung zur Assimilation oder gar als Beweis für deren Scheitern verstehen wollte, waren im nachhinein leicht zu finden, und zwar bereits in der Zeit des Kaiserreichs: Der Central Verein und der akademische Verein für jüdische Geschichte und Literatur wiesen ebenso wie die spezifischen, teilweise neuen Merkmale der jüdischen Familie oder der jüdischen Berufsstruktur darauf hin, daß, wie auch Scholem meinte, die deutschen Juden einen Prozeß der Dissimilation durchliefen. Dies nun bedeutet, daß die Kohärenz der Geschichte deutscher Juden nicht in der Assimilation, sondern im Wettbewerb zwischen Assimilation und Dissimilation bzw. in der Dialektik beider Phänomene liegt. Geht man als Historiker nicht von einer absoluten, vollständigen Assimilation aus und akzeptiert das Prinzip der Differenz, dann ergänzt und stabilisiert das Konzept der Dissimilation im Endeffekt den Assimilationsprozeß und trägt dazu bei, den Kern der Kohärenz dieser Geschichte klarzustellen.

So gesehen, ist die Übersteigerung des Begriffs Dissimilation, ist die jüdische Renaissance ebenfalls ein weiterer Beitrag zur Kohärenz der Geschichte der deutschen Juden: Als Pendant, Gegenbewegung oder auch Ergänzung des Assimilationsprozesses ist immer wieder die autonome jüdische Aktivität und das jüdische Bewußtsein - wie auch immer man dies definieren möchte - in die Kohärenz einzuordnen. Im Kaiserreich war es zunächst die rege Aktivität der neugegründeten jüdischen Vereine - Studentenverbindungen, Jugendbewegungen, Turn- und Sportvereine - mit ihrer Presse und ihren Veranstaltungen ${ }^{34}$, die auf ein neues Äquilibrium zwischen „Deutschsein" und "Jüdischsein" hinweisen mochte.

31 Alfred Kantorowicz, Liquidation der Judenfrage (1932).

$32 \mathrm{Vgl}$. Moshe Zimmermann, Geschichte der deutschen Juden 1914-1945 (München 1997) $1 \mathrm{f} ., 84-89$.

33 Michael Brenner, The Renaissance of Jewish Culture in Weimar Germany (New Haven 1996) 81 ; im folgenden zitiert: Brenner, Renaissance.

34 Jacob Borut, „Ein neuer Geist unter unseren Brüdern in Aschkenaz“. Der neue Weg des deutschen Judentums am Ende des 19. Jahrhunderts (hebr.; Jerusalem 1999). 
Während des Ersten Weltkrieges kam es dann gerade infolge der Begegnung mit den unter deutsche Besatzung geratenen polnischen Juden zum Erwachen eines "genuin" jüdischen Bewußtseins in Deutschland. Die Weimarer Republik gilt in der Forschung ohnehin als Impulsgeberin für eine präzedenzlose Kulturrenaissance $^{35}$ - das Jüdische Lehrhaus mit so prominenten Namen wie Martin Buber und Franz Rosenzweig oder die „Encyclopaedia Judaica“ mit Nahum Goldmann und Jakob Klatzkin als Herausgeber gelten als herausragende Beispiele. Sogar die jiddische Literatur erlebte damals eine Hochkonjunktur.

Paradox ist nun, daß es auch im „Dritten Reich“ eine Fortsetzung der „jüdischen Renaissancen“ (durchaus in der Mehrzahl) gab - der jüdische „Kulturbund“, die jüdische Presse und der jüdische Sport waren populär wie nie zuvor, nun allerdings infolge der diskriminierenden Gesetzgebung. Und doch ist hier nicht nur eine über das Jahr 1933 hinausreichende Kohärenz jüdischer Renaissancen zu konstatieren, sondern es geht auch um Erscheinungen, die gerade die assimilatorischen Motive zum Vorschein bringen. Wenn der Sportverein „Der Schild“ um Rekorde und Siege rang, ging es um die an „allgemeinen“ Maßstäben gemessene Ebenbürtigkeit deutscher Juden - eben getrennt und assimiliert zugleich.

Modernisierung und Assimilation, aber auch Dissimilation und jüdische Renaissance erweisen sich also als Komponenten der Kohärenz der Geschichte der deutschen Juden im ersten Drittel des 20. Jahrhunderts und danach. Jedoch steht in jeder Hinsicht der Anfang des zweiten Drittels vornehmlich eher unter dem Vorzeichen der Zäsur als der Kohärenz. Denn die Zäsur von 1943 wurde 1933 eingeleitet und war spätestens seit 1938 vorprogrammiert. Diese zehn Jahre dauernde Phase der Zäsur ist die Shoah der deutschen Juden. Weil eben das Ende schrittweise kam und diese Geschichte nicht allein unter der Überschrift des Antisemitismus zusammengefaßt werden kann, ist der Wandel der deutschen Judenheiten in Deutschland zwischen 1933 und der Nachkriegszeit eine besondere Herausforderung für den nach Kohärenz und Kontinuität suchenden Historiker, der sich der Erforschung des deutschen Antisemitismus widmet. Das ist spätestens seit dem Goldhagen-Streit allgemein bekannt.

Aber vielleicht schafft die Tiefe der Zäsur wiederum ein Paradox der Kohärenz: Nicht nur Renaissance und Dissimilation, sondern auch Zäsur und Renaissance sind untrennbare Begriffspaare. Die jüdische Renaissance beschäftigt erneut den Betrachter des deutschen Judentums nach 1945, insbesondere nach der Einwanderung von Juden aus den ehemaligen Ländern der Sowjetunion nach Deutschland in den 1990er Jahren: eine jüdische Renaissance in Deutschland? So fragt Michal Bodemann in seinem Buch "Gedächtnistheater" 36 und bezieht sich auf Sander Gilmans Stellungnahme zur "Wiedergeburt jüdischen Lebens in Deutschland“. Sollte man etwa die Kohärenz der jüdischen Geschichte im gesamten 20. Jahrhun-

35 Brenner, Renaissance.

36 Y. Michal Bodemann, Gedächtnistheater. Die jüdische Gemeinschaft und ihre deutsche Erfindung (Hamburg 1996) 42; im folgenden zitiert: Bodemann, Gedächtnistheater; vgl. auch Y. Michal Bodemann, In den Wogen der Erinnerung. Jüdische Existenz in Deutschland (München 2002). 
dert unter dem Titel „Zäsur und Renaissance“ zusammenfassen können? Als Prüfstein für das Bewußtsein von Kohärenz und Zäsur könnte man auch die Zukunftserwartungen der Juden selbst in Zeiten des Umbruchs, vor 1914 oder nach 1918 , einsetzen. Schaut man auf derartige Zukunftserwartungen von Juden gerade in Zeiten des Umbruchs oder der Krise - beim Aufstieg des Zionismus zu Beginn des 20. Jahrhunderts, während oder nach der Revolution von 1918 oder gar im „Entscheidungsjahr" 1932 -, so scheint die Haltung eher schwerfällig gewesen zu sein; man erwartete mehr Kohärenz und Kontinuität als Krise, man rechnete mit einer Stabilität der vorhandenen politischen und gesellschaftlichen Strukturen ${ }^{37}$.

\section{Kohärenzen nach 1943/45}

Nach Kriegsende galt zunächst Leo Baecks Spruch vom Ende des deutschen Judentums als axiomatisch. Arnold Zweig hatte bereits 1933 prognostiziert: „Die Zukunft der Juden [findet] im deutschen Geist keinen Halt mehr. "38 Es ist schwierig, die auf deutschem Boden nach 1945 lebenden Juden überhaupt als deutsche Juden zu definieren oder als kohärente Fortsetzung des deutschen Judentums der Vorkriegszeit zu verstehen. Die wenigen deutschen Juden, die das „Dritte Reich“ überlebt hatten, sowie die zurückkehrenden deutschen Juden machten nicht nur statistisch einen winzigen Bruchteil des früheren deutschen Judentums aus. Sie waren auch eine Minderheit in der vornehmlich aus DP-Juden sich organisierenden jüdischen Bevölkerung im Land. Als in den 1990er Jahren der Umfang der jüdischen Bevölkerung in Deutschland dramatisch von rund 30000 auf 100000 Juden stieg, offenbarte sich die Zäsur noch deutlicher: Es waren nun vornehmlich Juden aus den Ländern der Sowjetunion, die der jüdischen Gemeinschaft in Deutschland ganz andere Impulse zuführten als die Einwanderer aus dem Osten vor 1933.

Was dennoch eine gewisse Kohärenz aufwies, war weniger die Tatsache, daß Juden in beiden deutschen Staaten lebten, sondern vielmehr der Ort, den Juden in der kollektiven Erinnerung beider Gesellschaften einnahmen. Zunächst - bis kurz nach Gründung der Bundesrepublik Deutschland - äußerte sich die Kohärenz in der Fortsetzung von antisemitischen Vorurteilen, wie sich aus OMGUS-Umfragen ergibt ${ }^{39}$. Dann aber kam es allmählich und seit Ende der 1960er Jahre mit zu-

37 Vgl. Moshe Zimmermann, Das Gesellschaftsbild der deutschen Zionisten vor dem Ersten Weltkrieg, in: Truma 1 (Wiesbaden 1987) 139-158. Moshe Zimmermann, , Die aussichtslose Republik' - Zukunftsperspektiven der deutschen Juden vor 1933, in: Menora. Jahrbuch für deutsch-jüdische Geschichte (München, Zürich 1990) 152-183; Moshe Zimmermann, Zukunftserwartungen deutscher Juden im ersten Jahr der Weimarer Republik, in: Archiv für Sozialgeschichte (Bonn 1997) 265-280.

38 Arnold Zweig, Bilanz der deutschen Judenheit 1933 (Berlin 1998) 230; im folgenden zitiert: Zweig, Bilanz.

39 Anna J. Merritt, Richard L. Merritt, Public opinion in occupied Germany: The Omgus surveys, 1945-1949 (Urbana 1970). 
nehmender Deutlichkeit zu einer „Aufarbeitung“ der deutsch-jüdischen Vergangenheit, zu einer geistigen „Wiedergutmachung“. In den „Wochen der Brüderlichkeit“, in Schrift und Literatur versuchte man in einem „Deutschland ohne Juden" ${ }^{40}$ wenigstens die Erinnerung als Ersatz für die vertriebenen und ermordeten deutschen Juden und für die fehlende jüdische Präsenz in der Bundesrepublik am Leben zu erhalten. Diese nichtjüdische Erinnerung an die deutschen Juden zielt vor allem auf die deutsch-jüdische Geschichte der Zeit vor dem „Dritten Reich“, während sich die kollektive, aktivierte Erinnerung der neuen deutschen Juden nahezu ausschließlich auf die Shoah zu konzentrieren scheint und gerade die frühere deutsch-jüdische Geschichte ausblendet. So ergibt sich bei deutschen und vielen anderen Juden ein Bruch des Erinnerungsprozesses in Parallele zur Zäsur von 1943 in der jüdischen Geschichte als solcher ${ }^{41}$. Durch Kontakte zu den „ehemaligen jüdischen Mitbürgern", die von deutschen Städten gepflegt werden, oder durch die Gründung von jüdischen Museen und Instituten für jüdische Geschichte bemüht sich die deutsche Gesellschaft um eine ungewöhnliche Kohärenz, mit der die zwölf schrecklichen Jahre überbrückt werden sollen, wodurch allerdings die Zäsur dialektisch um so mehr unterstrichen wird ${ }^{42}$. Literarische Werke wie Ralf Giordanos „Die Bertinis“ oder Victor Klemperers Tagebücher konfrontieren ihre Leser mit dem Phänomen der Assimilation und dem Problem des AuBenseiters als Insider und lassen diese Bereiche neue Aktualität gewinnen. Sie schaffen eine gedankliche Kohärenz über das Jahr 1943 hinaus und verdeutlichen zugleich die Tiefe der durch die NS-Zeit entstandenen Zäsur.

Auch in der DDR spielten Juden in der kollektiven Erinnerung eine, wenn auch begrenzte Rolle. Zynisch bemerkte hierzu der Autor eines Buches über die Erinnerung der DDR an den Holocaust: „Tote Juden waren für ostdeutsche Belange nürzlicher" ${ }^{43}$; und auch das nur dort, wo überhaupt Juden (nicht Zionisten) thematisiert werden durften. Eine starke Präsenz von Juden (nach unterschiedlicher Definition) im Geistesleben der DDR - Stefan Heym, Stephan Hermlin, Arnold Zweig, Anna Seghers, Wolf Biermann u. a. - schien auf eine diesbezügliche Kontinuität über das Jahr 1945 hinaus eher in der DDR als in der Bundesrepublik hinzuweisen ${ }^{44}$. Mancher Rückkehrer hoffte, gerade in der DDR den direkten Anschluß an die sozialistische Vergangenheit vor $1933 \mathrm{zu}$ finden. Aber auch hier überschattete die Zäsur eindeutig die Kohärenz ${ }^{45}$. In beiden Teilen Deutschlands

40 Bernt Engelmann, Deutschland ohne Juden. Eine Bilanz (München 1970).

$41 \mathrm{Vgl}$. Lynn Rapaport, Jews in Germany after the Holocaust. Memory, Identity and JewishGerman Relations (Cambridge 1997) 27-38.

42 Vgl. Erica Burgauer, Zwischen Erinnerung und Verdrängung, Juden in Deutschland nach 1945 (Hamburg 1993); Andreas Nachama, Julius Hans Schoeps (Hrsg.), Aufbau nach dem Untergang (Berlin 1992); Michael Cohn, The Jews in Germany 1945-1993. The Building of a Minority (Westport, Conn., 1994).

43 Thomas C. Fox, Stated Memory. East Germany and the Holocaust (Rochester, NY. 1999)

53-60; im folgenden zitiert: Fox, Stated Memory; Bodemann, Gedächtnistheater 100-112.

44 Angelika Timm, Jewish Claims against East Germany (Budapest 1997) $29 \mathrm{ff}$.

45 Vgl. Fox, Stated Memory 79-93. 
wurde das Bewußtsein für die Tiefe der Zäsur gerade durch die Überwindung der Verdrängung immer größer.

Der mit fortschreitender Erinnerungsarbeit immer tiefere Dimensionen erreichende Schock konnte zwei exterritoriale, d.h. außerhalb Deutschlands gelegene Wege der Fortsetzung einer Kohärenz nicht blockieren. Juden als Träger bzw. Vertreter der bürgerlichen deutschen Kultur konnten gerade wegen der Vertreibung eine besondere Kohärenz schaffen. Arnold Zweig hatte bereits 1933 dieses Phänomen in aller Unzufriedenheit geschildert: „Auf der ganzen Erde haben Juden das Deutschtum verbreitet, überschätzt, ja angebetet ... Überall wo man sie aufnahm, als die Hitlerei sie hinausgeworfen, gaben sie zu Klagen Anlaß, weil sie einfach nicht aufhören konnten, die fremde Welt mit den Maßen ihres deutschen Zentralitätsaffektes zu bewerten ... Man sieht, es ist ... das, Und es soll am deutschen Wesen noch einmal die Welt genesen“, das die deutschen Juden überallhin begleitet. Ja, sie verstehen sich selbst im neuen erzwungenen Zustand als ,Auslandsdeutsche“."46

Nach der Katastrophe schrieb Paul Celan: „Unverloren blieb inmitten der Verluste dies eine: die Sprache ... sie ging durch dieses Geschehen, ging hindurch und durfte wieder zutage treten, ,angereichert" von all dem. " 47 Für diejenigen, die in der deutschen Sprache beheimatet waren, hat dieses Bild eine besondere Aussagekraft. Die deutsche Sprache, von deutsch-jüdischen Emigranten/Vertriebenen benutzt, wurde eine Brücke über den Abgrund, ein Weg zur Kohärenz. Und mit der Sprache waren es andere typisch deutsch-jüdische Merkmale, die viel mit den sogenannten deutschen Tugenden zu tun hatten, sowie die Zugehörigkeit zu deutsch-jüdischen Netzwerken. Sogar die Erinnerung an die deutsch-jüdische Vergangenheit wurde in den Ländern des Exil institutionalisiert, zum Beispiel in Form des Leo-Baeck-Instituts, das in drei Zentren des Exils wirkt - in London, New York und Jerusalem.

Das deutsche Judentum als Kulturwerk konnte sich, so gesehen, über das Jahr 1945 hinaus „retten“, unabhängig vom politischen und physischen Schicksal im „Dritten Reich“. Klar und trotzig formulierte George Mosse das Paradox von der Kohärenz außerhalb Deutschlands jenseits des Jahres 1945 mit den Worten: „It was the German-Jewish Bildungsbürgertum which, more than any other single group, preserved Germany's better self across dictatorship, war, holocaust and defeat." 48

Tatsächlich setzten die deutsch-jüdischen Emigranten in den USA, in Südamerika und in Israel in kultureller Hinsicht das fort, was die Assimilation vor 1933 geschaffen hatte. Daß dies in den Ländern einer „Ersatz-Diaspora“ geschehen konnte, verwundert wenig. Überraschend ist diese Tendenz vielmehr in dem Land, das angeblich von der Assimilation, vom Wesen der Diaspora und von der deutschen Tradition bewußt und trotzig Abschied genommen hatte - Palästina/

46 Zweig, Bilanz $228 \mathrm{f}$.

47 Paul Celan, „Die Sprache blieb unverloren“ (1958).

48 Mosse, Beyond Judaism 82. 
Israel. Eine Kohärenz im kulturellen, wirtschaftlichen und sozialen Bereich vor und nach der Emigration - in der zweiten Makkabiade von 1935 war das Dressurreiten bereits eine anerkannte Disziplin - war zu erwarten gewesen ${ }^{49}$. Doch selbst in der Politik des Zionismus in der "nationalen Heimstätte“ konnte man eine Kontinuität erkennen zwischen der kompromißbereiten Haltung der Mehrheit der deutschen Zionisten gegenüber den Arabern und der Haltung der Gruppe Brit Shalom (Friedensbund), die für eine bi-nationale Lösung des arabisch-jüdischen Konflikts eintrat, oder der Haltung der von deutsch-jüdischen Israelis nach dem Vorbild der deutschen Fortschrittspartei gegründeten Progressiven Partei in Sachen Verfassung und Bürgerrechte.

Die Kohärenz, die die Kombination „deutsch-jüdisch“ nach 1945 außerhalb Deutschlands anbietet, führt allerdings graduell doch zu einer Zäsur, und zwar in den 1970er Jahren. Paradoxerweise zeigt sich hier eine Parallelität der Zäsuren zwischen der deutschen Nachkriegsgesellschaft im allgemeinen, auf die die anderen Beiträge in diesem Band mit Nachdruck aufmerksam machen, und der deutsch-jüdischen Gesellschaft nach der Auswanderung. Die Gemeinsamkeit ist naturgemäß durch die Entwicklung der Generationen bedingt: Hier wie dort geht es um das Ausscheiden der Generation, die das "Dritte Reich" als Erwachsene erlebt hatte. Die oben erwähnte deutsch-jüdische Tradition, Kultur und Gesellschaft in den USA, in Israel und Südamerika, die im Exil fortleben konnte, verschwindet mit dem Wechsel zur nächsten Generation. Der Lebensstil deutsch-jüdischer Emigranten ging in den 1970er Jahren tatsächlich seinem Ende entgegen, wie Steven Lowenstein im Epilog zum vierten und letzten Band der vom LeoBaeck-Institut herausgegebenen deutsch-jüdischen Geschichte in der Neuzeit aus der Perspektive der 1990er Jahre bemerkte ${ }^{50}$. Da die Geschichte des Judentums in Deutschland ihren Rahmen und ihre Koordinaten nicht nur in der deutschen, sondern auch in der universal-jüdischen Geschichte findet, sind Fragen der Kohärenz und der Zäsuren erstens mit den verschiedenen Definitionen von Judentum, zweitens mit den allgemeinen Tendenzen der jüdischen Gesellschaft und drittens mit dem Gang der jüdischen Historiographie eng verbunden. Schaut man auf das Judentum als Religion oder Religionsgemeinschaft, zeigt sich der Einfluß des "deutschen Modells“ sehr konsequent: Die jüdisch-religiöse Reformbewegung, die von Deutschland ausging und sich in Amerika seit Mitte des 19. Jahrhunderts verbreiten und entfalten konnte, erlebte auch zwischen 1933 und 1945 keine Existenzkrise. Schaut man auf die Prozesse der Assimilation, Emanzipation und Integration, so wird deutlich, daß sie außerhalb Deutschlands auch nach 1945 fortgesetzt wurden - die national-jüdische Alternative konnte selbst auf dem Hintergrund des verheerenden Scheiterns des deutschen Experiments der Assimilation

49 Einen guten Uberblick verschafft Yoav Gelber, Neue Heimat (hebr.; Jerusalem 1990); siehe auch: Gideon Greif, Colin McPherson, Laurence Weinbaum (Hrsg.), Die Jeckes: Deutsche Juden aus Israel erzählen (Köln 2000).

50 Stephen M. Lowenstein, Epilog: Die deutsch-jüdische Diaspora, in: Avraham Barkei, Paul Mendes-Flohr, Deutsch-jüdische Geschichte in der Neuzeit, Bd. 4 (München 1998). 
die jüdische Welt nicht monopolisieren. Doch im engeren Rahmen der deutschen, ja sogar der europäischen Geschichte muß wohl zusammenfassend die traumatische Krise der Jahre 1943-1945 in den Mittelpunkt der Erwägungen rücken, selbst wenn die Kohärenzen der deutsch-jüdischen Geschichte weitreichender sein mögen, als man traditionell vermutet. 
